\title{
Environmental Signals Modulate the Expression of an Indole-3-Acetic Acid Biosynthetic Gene in Erwinia herbicola
}

\author{
Maria T. Brandl and Steven E. Lindow \\ Plant and Microbial Biology, 111 Koshland Hall, University of California, Berkeley 94720, U.S.A. \\ Received 12 November 1996. Accepted 3 February 1997.
}

\begin{abstract}
The ipdC gene of Erwinia herbicola strain 299R encodes an indolepyruvate decarboxylase involved in the biosynthesis of indole-3-acetic acid (IAA). Transcriptional fusions of ipdC to an ice nucleation reporter gene (inaZ) were used to study the expression of ipdC in vitro and in situ on plants. ipdC was expressed only at low levels in liquid media and independently of factors such as richness of the medium, pH, nitrogen availability, the presence of L-tryptophan or oxygen, and growth phase of the culture. However, the transcriptional activity of $i p d C$ increased approximately 18-fold under low solute and matric potentials in culture. ipdC was also induced 32-fold on leaves of bean and tobacco and 1,000-fold on pear flowers. This is the first report of the plant-inducible transcription of a bacterial IAA biosynthetic gene. It strongly supports the role of ipdC, and thus that of the indole-3-pyruvic acid pathway, in IAA biosynthesis by strain 299R in situ. The plant induction and apparent regulation of $i p d C$ by low water availability indicate that this gene, and presumably IAA synthesis, are involved in a response to conditions encountered by $E$. herbicola in its natural habitat on leaves.
\end{abstract}

Additional keywords: auxin, epiphyte, osmoregulated, Pantoea agglomerans, tryptophol, water stress.

The biosynthesis of indole-3-acetic acid (IAA) in culture media amended with L-tryptophan (TRP) is prevalent among strains of Erwinia herbicola (Pantoea agglomerans) isolated from plant surfaces (E. M. Clark and S. E. Lindow, unpublished data). Applications of IAA-producing strains of E. herbicola to pear trees during flowering increased the severity of fruit russet (Lindow 1987), a major problem in the production of pome fruits. Presumably, the secretion of bacterial IAA onto the fruit surface altered the normal epidermal cell development of the fruits. E. herbicola strain 299R produces IAA by the indole-3-pyruvic acid (IPyA) pathway, and although many routes for IAA synthesis have been described in bacteria, no evidence was found for the existence of alternative pathways in this strain (Brandl et al. 1996). The ipdC gene, which encodes the enzyme responsible for the decarboxylation of IPyA to yield indole-3-acetaldehyde (IAAld), the immediate precursor of IAA, was cloned from E. herbicola strain

Corresponding author: S. E. Lindow; Tele.: 510-642-4175; Fax: 510642-4995; E-mail: icelab@violet.berkeley.edu
299R and its nucleotide sequence determined (Brandl and Lindow 1996). The insertional inactivation of ipdC resulted in a substantial reduction in the ability of strain 299R to synthesize IAA in culture, confirming the role of the IPyA pathway in the production of IAA in this strain (Brandl and Lindow 1996). The ecological significance of IAA production to the bacteria that synthesize it, and the potential effects of exogenous IAA on the plants that they colonize, would be supported if it were demonstrated that bacterial IAA production occurs while bacteria are on plants. To date, much of the evidence for the importance of IAA production in plant-microbe interactions has been inferred from the attenuation of symptoms exhibited by plants infected with IAA mutants of gall-forming bacteria (Yamada 1993). Although several additional studies have reported the presence of IAA in culture supernatants of various microorganisms, there is little evidence that these synthesize IAA in their natural habitats. Studies of the differential expression of bacterial genes in various habitats have proven useful to better understand how bacteria have evolved to exploit these sites. For example, substantial phenotypic differences have been observed in an epiphytic strain of Pseudomonas syringae when grown in culture and on plants (Wilson and Lindow 1993). These differences in the behavior of cultured cells from that of cells recovered from plants were associated with different patterns of gene expression; about $2 \%$ of the genes of $P$. syringae are apparently expressed when cells are on plants but are not expressed in culture (Cirvilleri and Lindow 1994). In addition, many genes from phytopathogenic bacteria are expressed in a habitat-specific pattern, and several are inducible by plant signal molecules (Lindow 1995). Knowledge of the in situ transcriptional activity of bacterial genes involved in IAA synthesis is completely lacking. To gain insight into the ecological significance of IAA synthesis in epiphytic bacteria, we used fusions of a promoterless ice nucleation gene (inaZ) (Lindgren et al. 1989) to $i p d C$ to study the transcriptional regulation of ipdC in E. herbicola under various culture conditions and on plant surfaces.

\section{RESULTS}

ipdC induction on plants.

The ice nucleation activity of E. herbicola 299MX149, which contains a transcriptional fusion of ipdC to inaZ, was 1,000-fold higher after incubation on tobacco leaf surfaces than when cells were grown in minimal A medium (activity of 
inoculum at $0 \mathrm{~h}$ ) (Fig. 1). Due to the exponential relationship between ice nucleation activity and abundance of ice nuclei (Lindgren et al. 1989), this increase in ice nucleation activity on tobacco leaves represents a 32-fold increase in expression of ipdC. The induction of ipdC occurred at least as early as 10 $\mathrm{h}$ after inoculation with 299MX149, when cells were growing exponentially. Significantly, the ice nucleation activity remained high even after the rate of cell multiplication decreased (Fig. 1). Similar observations were made on young bean plants (data not shown).

Expression of ipdC in strain 299MX149 was detected on pear $35 \mathrm{~h}$ after inoculation of opened flowers, when the cells were actively growing (Fig. 2A and B). At maximum expression of ipdC, the ice nucleation activity of 299MX149 was $10^{6}$-fold greater than in the minimal A broth culture used for preparation of the inoculum (activity at $0 \mathrm{~h}$ ) (Fig. 2A), which is equivalent to a 1,000-fold increase in transcriptional activity of ipdC. In contrast, the control strain 299R(pJEL1703), which carries ice $C$ expressed from its native promoter, showed only a relatively small increase in ice nucleation activity (89-fold) (Fig. 2A).

Because the secretion of nectar in pear flowers appeared visually to progressively decrease during the period of bacterial colonization, the dryness of the nectary was quantified. As reflected by the electrical resistance across the nectary, the flowers dried very rapidly after about 3 days following opening (Fig. 3). Since the flowers had been open on average for about 2 days at the time of inoculation (Fig. 2A), this period of increasing dryness thus corresponds to the time at which ipdC reached high levels of expression on pear flowers.

Factors regulating transcriptional activity of ipdC in vitro.

Effect of culture conditions. The ice nucleation activity of 299MX149 cultured in minimal A, Luria-Bertani (LB), and

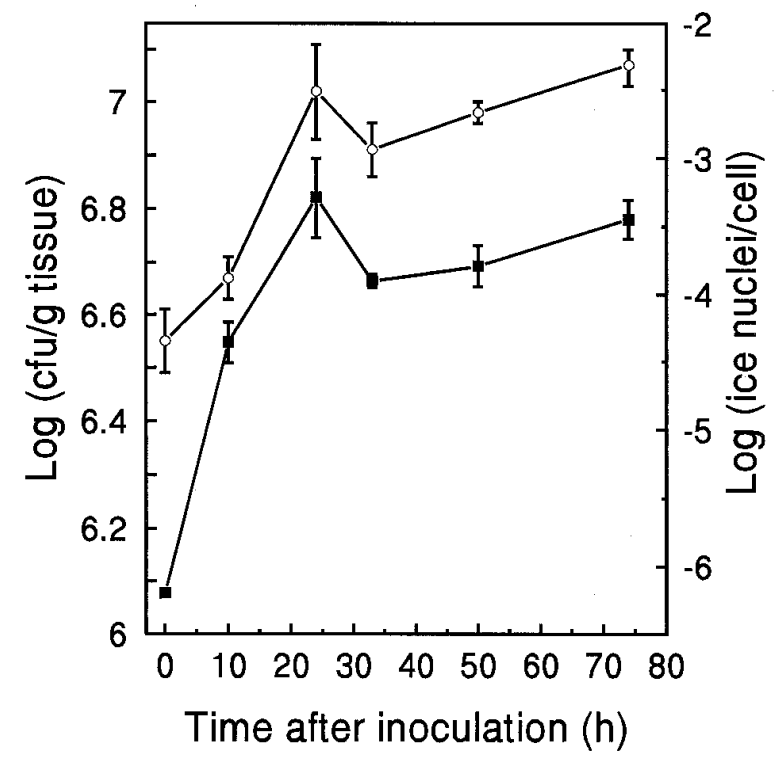

Fig. 1. Expression of ipdC on tobacco plants. Ice nucleation activity (ש and population size (O) of Erwinia herbicola 299MX149 on tobacco leaves were determined as a function of time after inoculation. Ice nucleation activity at time of inoculation $(0 \mathrm{~h})$ is that of the inoculum suspension. Values represent the mean and standard error of the mean.
King's B broth was relatively low, ranging from about $10^{-7.3}$ to $10^{-6.6}$ ice nuclei per cell. No significant change in ice nucleation activity was observed at any stage of growth of 299MX149 in these culture media. The addition of TRP (the main precursor to IAA in E. herbicola) to the culture medium did not affect the expression of ipdC $\left(10^{-6.87}\right.$ ice nuclei per cell). Because the production of IAA is increased in nitrogenlimiting culture conditions (Brandl et al. 1996), the ice nucleation activity of 299MX149 was compared in conditions of low and high nitrogen availability. The levels of ice nucleation activity in minimal A medium containing only $0.01 \%$ ammonium sulfate (low nitrogen availability) $\left(10^{-6.77}\right.$ ice nuclei per cell) were the same as in regular minimal A medium $(0.1 \%$ ammonium sulfate; high nitrogen availability) $\left(10^{-6.87}\right.$ ice nuclei per cell). Moreover, the substitution of various carbon sources, including sucrose, fructose, galactose, and succinate, for the glucose in minimal A broth (lacking sodium citrate) did not affect the expression of ipdC, which remained low in
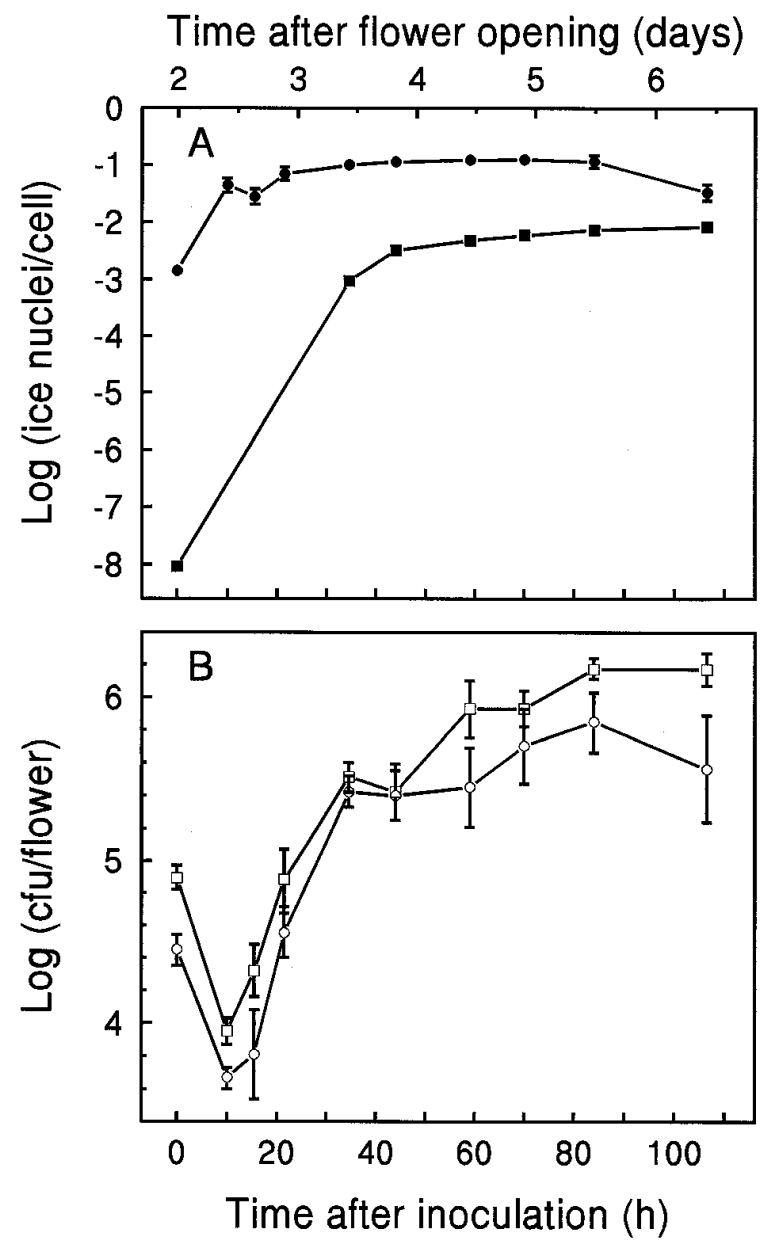

Fig. 2. Expression of ipdC and growth of Erwinia herbicola strains on pear flowers in the greenhouse. Ice nucleation activity (A) and population size (B) of strain 299MX149 (squares) and strain 299R pJEL1703 (circles) on pear flowers were determined as a function of time after inoculation. Ice nucleation activity at time of inoculation $(0 \mathrm{~h})$ is that of the inoculum suspension; that at 10,15 , and $21 \mathrm{~h}$ after inoculation is below the detection limit. Values represent the mean and standard error of the mean. 
all cases $\left(10^{-6}\right.$ to $10^{-7}$ ice nuclei per cell). In contrast, the ice nucleation activity of 299MX149 grown on minimal A and LB agar was about 350 - to $10^{4}$-fold higher than that of 299MX149 grown in broth. The levels of expression on agar plates were thus comparable to those detected on plants. No significant differences in the ice nucleation activity of the control strain 299R(pCPPICE), which carries ice C expressed from its native promoter on the vector $\mathrm{pCPP} 8$, were observed under any of the tested culture conditions.

Because E. herbicola is a facultative anaerobe, the effect of oxygen availability on ipdC expression was also investigated. The ice nucleation activities of 299MX149 grown on minimal A agar under either aerobic or anaerobic conditions were not significantly different.

In order to test the effect of $\mathrm{pH}$ on the expression of ipdC, strain 299MX149 was grown in minimal A medium buffered with potassium phosphate at various $\mathrm{pH}$ values (Sambrook et al. 1989). The apparent expression of ipdC was low at all $\mathrm{pH}$ values; ice nucleation activity of 299MX149 cultured in TRPamended minimal A broth of $\mathrm{pH} 5.8,6.4,7.2$, and 8.0 was $10^{-6.63}, 10^{-7.60}, 10^{-7.65}$, and $10^{-5.85}$ ice nuclei per cell, respectively.

Effect of osmotic stress. The observation that ipdC expression in E. herbicola on pear blossoms increased upon the drying of the nectary led to investigation of the effect of osmotic stress on the transcriptional activity of ipdC-inaZ fusions. The ice nucleation activity of strain 299MX149 cultured in minimal A broth supplemented with various osmolytes increased greatly with decreasing solute potential of the medium. Because the growth rate of the cells was dramatically reduced at $-3.7 \mathrm{MPa}$, ice nucleation activity was not tested in a medium of osmotic pressure lower than $-2.7 \mathrm{Mpa}$. The ice nucleation activity of 299MX149 was 5,400-fold higher at a solute potential $\left(\psi_{\mathrm{s}}\right)$ of $-2.7 \mathrm{MPa}$ generated by the presence of $\mathrm{NaCl}$ in minimal A medium than in regular mini-

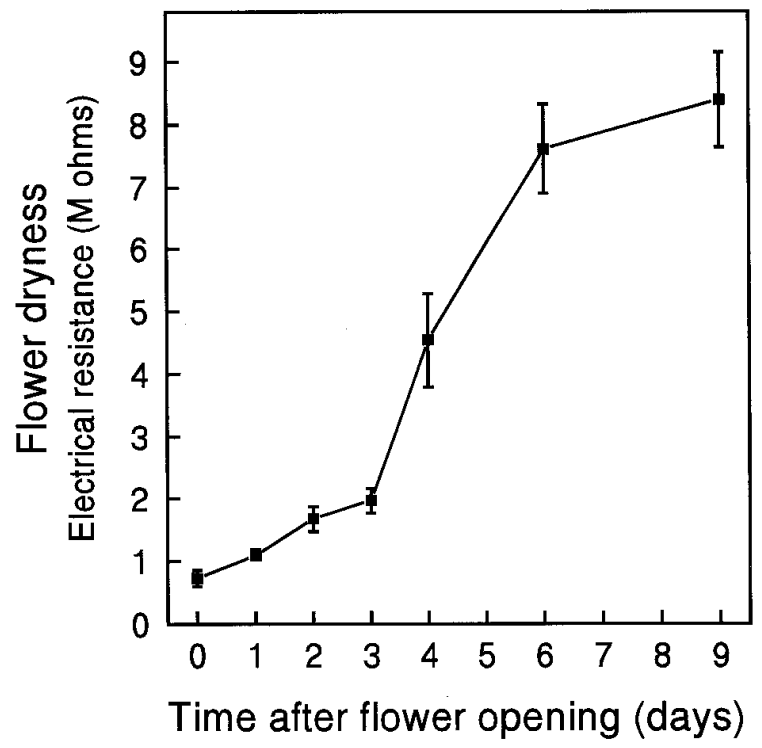

Fig. 3. Estimates of water availability on pear flowers made by measurement of electrical resistance across the nectary as a function of time after opening of the flowers. Values represent the mean and standard error of the mean of 10 flower samples from four different trees placed in the greenhouse. mal A broth $\left(\psi_{\mathrm{s}}=-0.7 \mathrm{Mpa}\right)$ (Fig. 4), reflecting a 73-fold increase in transcriptional activity of ipdC upon exposure of 299MX149 to hyperosmotic conditions. $\mathrm{NaCl}, \mathrm{KCl}$, sorbitol, and $\beta$-lactose all elicited increased transcription of ipdC in a similar manner (Fig. 4). The control strain 299R(pCPPICE) expressed only slightly higher levels of ice nucleation activity (about 10-fold on average) when grown under conditions of high osmolarity.

To determine the rate at which ipdC transcription responded after osmotic shock, the change in ipdC expression with time after transfer of 299MX149 from minimal A medium to minimal A medium containing $1.28 \% \mathrm{NaCl}\left(\psi_{\mathrm{s}}=-1.7 \mathrm{MPa}\right)$ was studied. Maximum induction of ipdC was detected within $3.5 \mathrm{~h}$ after osmotic shock (Fig. 5). Increased transcription of ipdC thus occurred during the exponential phase of growth. In contrast, the expression of ipdC remained at low levels in minimal A medium in the absence of $\mathrm{NaCl}$. Strain 299MX149 exhibited a growth rate similar to that of the parental strain in TRP-amended minimal A medium adjusted to solute potentials of $-1.2,-1.7$, and -2.7 with $\mathrm{NaCl}$ (data not shown).

Effect of matric stress. To determine if low water potential per se rather than osmotic stress triggered ipdC induction, the effect of high matric potential $\left(\psi_{\mathrm{m}}\right)$ on ipdC expression was investigated in culture. Polyethylene glycol with an average molecular weight of 4,000 (PEG 4000), which is impermeant to cell walls and membranes (Harris 1981), was added at various concentrations to minimal A broth to reduce the water availability in the culture medium. The matric potential of regular minimal A broth was considered to be approximately 0 . The apparent growth rate of strains 299R, 299MX149, and $299 \mathrm{R}$ (pCPPICE), was only slightly reduced at $\psi_{\mathrm{m}}=-0.5 \mathrm{Mpa}$, with a greater decrease at $-0.75 \mathrm{MPa}$; no growth was observed at $-1.0 \mathrm{MPa}$ (data not shown). A decrease in matric potential to $-0.2 \mathrm{MPa}$ was sufficient to increase transcription of ipdC

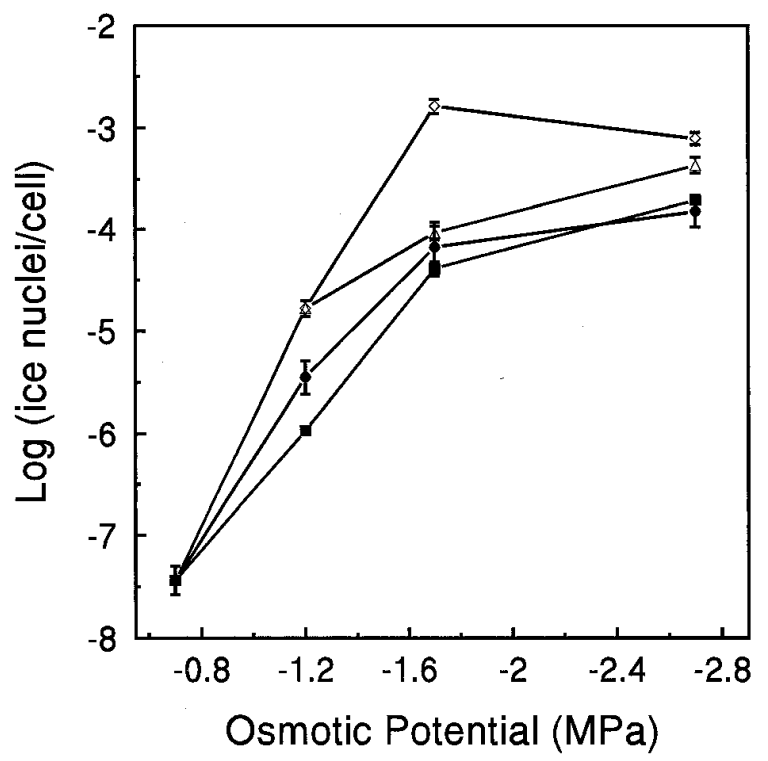

Fig. 4. Effect of osmotic stress on expression of ipdC. Ice nucleation activity of Erwinia herbicola $299 \mathrm{MX} 149$ is shown as a function of solute potential generated by the presence of $\mathrm{NaCl}(\boldsymbol{\square}), \mathrm{KCl}(\bullet)$, sorbitol $(\triangle)$, or $\beta$-lactose $(\diamond)$ in the culture medium. Each value represents the mean and standard error for two replicate cultures. 
(Fig. 6). Maximum induction of ipdC occurred at $-0.5 \mathrm{MPa}$; at this matric potential, a 1,300-fold increase in ice nucleation activity of $299 \mathrm{MX} 149$, which is equivalent to a 36 -fold increase in transcriptional activity of ipdC, above that in unamended minimal medium was observed. No significant increase in ice nucleation activity of the control strain 299R(pCPPICE) was detected under the same matric potential conditions tested as described above.

\section{Production of tryptophol and IAA under osmotic stress.}

Since the ability of strain 299R to synthesize IAA, IAAld, and tryptophol (TOL; an indole derivative that is produced reversibly from IAAld) in culture was greatly reduced by insertional inactivation of ipdC (Brandl and Lindow 1996), we tested whether the levels of these compounds were related to the estimated rate of ipdC transcription under water stress conditions. The effect of low water availability on the production of IAA and TOL by strain 299R was determined by quantification of these compounds in cultures containing different amounts of $\mathrm{NaCl}$. Since the presence of high concentrations of $\mathrm{NaCl}$ reduced cell growth rates, the quantities of TOL and IAA detected in the culture supernatant were normalized for the number of cells, to take into account differences in the cell concentration of the cultures. The production of TOL increased in response to hyperosmotic conditions, with a 2.0- and 2.6-fold higher level at osmotic pressures of -1.2 and $-1.7 \mathrm{MPa}$, respectively, than in unamended minimal A medium (Table 1). A higher level of IAA synthesis was also observed as a consequence of incubation at a solute potential of $-1.2 \mathrm{MPa}$, but no further increase occurred at lower solute potentials.

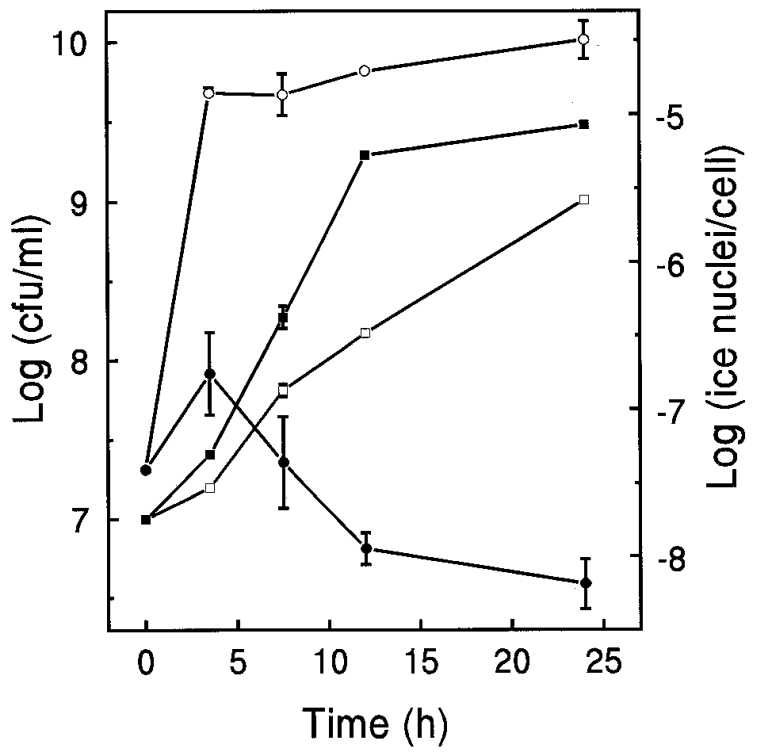

Fig. 5. Time course expression of ipdC in L-tryptophan (TRP)-amended minimal A broth in the absence (closed symbols) or presence of $1.28 \%$ $\mathrm{NaCl}\left(\psi_{\mathrm{s}}=-1.7 \mathrm{MPa}\right)$ (open symbols) after transfer from TRP-amended minimal A broth. Ice nucleation activity (circles) and cell concentration of Erwinia herbicola 299MX149 (squares) were determined as a function of time after inoculation. Values represent the mean and standard error for two replicate cultures.

\section{DISCUSSION}

The study of the transcriptional activity of ipdC-inaZ fusions in 299MX149 under various growth conditions revealed that ipdC is expressed at very low levels in broth culture, and independently of the richness, nitrogen content, and $\mathrm{pH}$ of the medium, $\mathrm{O}_{2}$ availability, and the presence of TRP, the main IAA precursor in E. herbicola. Unlike expression of certain hrp genes of $P$. syringae pv. phaseolicola and Xanthomonas campestris pv. campestris (Rahme et al. 1992; Schulte and Bonas 1992), and the avrB gene of $P$. syringae pv. glycinea (Huynh et al. 1989), which are also plant inducible, the expression of ipdC was not affected by the type of carbon source in the culture medium. However, ipdC transcription increased 32- and 1,000-fold when 299MX149 was grown on tobacco and bean leaves, and pear blossoms, respectively. The comparatively small increase in the ice nucleation activity of the control strain 299(pJEL1703) while on plants indicates that high levels of ice activity did not result simply from an effect of the plant environment on the production of ice nucleation

Table 1. Production of tryptophol (TOL) and indole-3-acetic acid (IAA) in cultures of Erwinia herbicola $299 \mathrm{R}$ under hyperosmotic conditions ${ }^{\mathrm{a}}$

\begin{tabular}{|c|c|c|}
\hline \multirow[b]{2}{*}{ Osmotic potential $(\mathrm{MPa})^{\mathrm{b}}$} & TOL & IAA \\
\hline & \multicolumn{2}{|c|}{$\overline{(\mu \mathrm{g} / 10} \overline{9}$ cells $)$} \\
\hline-0.7 & $0.67 \pm 0.19$ & $3.06 \pm 1.09$ \\
\hline-1.2 & $1.30 \pm 0.21$ & $4.85 \pm 1.13$ \\
\hline-1.7 & $1.78 \pm 0.07$ & $2.20 \pm 0.03$ \\
\hline-2.7 & $0.10 \pm 0.04$ & $0.05 \pm 0.03$ \\
\hline
\end{tabular}

${ }^{a}$ Each value is the mean and standard error for two replicate cultures. Similar results were obtained in two separate experiments.

b The solute potential of L-tryptophan-supplemented minimal A medium was adjusted by the addition of $\mathrm{NaCl}$ at concentrations described in Material and Methods.

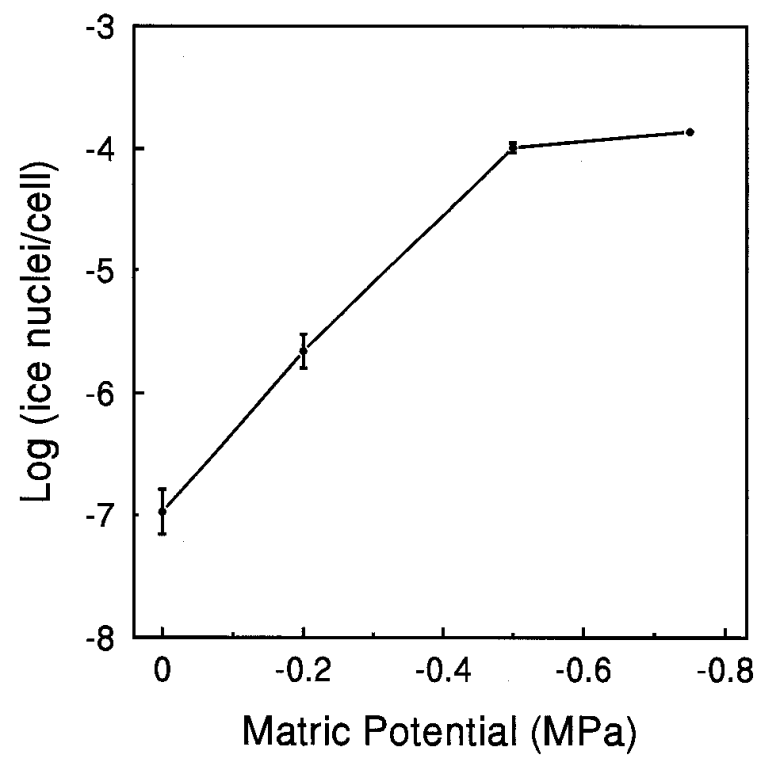

Fig. 6. Effect of matric potential on expression of ipdC. Ice nucleation activity of Erwinia herbicola 299MX149 is shown as a function of matric potential conferred by presence of PEG 4000 in the culture medium. Values represent the mean and standard error for two replicate cultures. 
sites in the bacteria, but rather from increased transcriptional activity of ipdC. It thus appears that a signal from the physicochemical environment of plant surfaces is involved in the regulation of ipdC transcription and presumably of IAA synthesis. The insertional inactivation of ipdC resulted in a significant decrease in IAA production by strain 299R in culture (Brandl and Lindow 1996). It also abolished the ability of this strain to inhibit radish root elongation in a laboratory root bioassay for IAA (M. T. Brandl and S. E. Lindow, unpublished data). This observation, along with the increased transcriptional activity of $i p d C$ on plants, suggests a role for ipdC and hence the IPyA pathway in the production of IAA by E. herbicola on plant surfaces and not solely under culture conditions. This is the first study that demonstrates the plant-inducible transcription of a bacterial IAA biosynthetic gene in situ.

The low expression of ipdC in culture may be the reason why significant amounts of IAA are produced by E. herbicola only in the presence of high tryptophan concentrations in culture (Brandl et al. 1996). Presumably, considerable amounts of IAA could be synthesized in cells containing very low levels of indolepyruvate decarboxylase only when high quantities of TRP, the main precursor in the IAA pathway, are available. In contrast, higher levels of indolepyruvate decarboxylase may facilitate the synthesis of IAA on plants, where tryptophan availability is apparently low (Morgan and Tukey 1964; Weibull et al. 1990). Indeed, the increased amounts of this enzyme may enhance IAA synthesis by depleting indolepyruvate, the product resulting from the deamination of tryptophan in the first reaction of the IAA pathway, thus ensuring the continued channeling of tryptophan into the IAA biosynthetic pathway. It is significant that the transcription of ipdC is not induced by tryptophan. The presence of tryptophan, however, induces the expression of a gene encoding an aromatic aminotransferase that deaminates tryptophan to produce indolepyruvate, the substrate of ipdC (E. M. Clark and S. E. Lindow, unpublished data).

The positive correlation between the dryness of the surface of pear flowers and the ice nucleation activity of 299MX149 suggested that water potential may govern expression of ipdC, and potentially IAA synthesis. The 73-fold increase in the transcriptional activity of ipdC-inaZ fusions in minimal A broth of high osmolarity supports this hypothesis. The finding that sorbitol and lactose elicited a response similar to that of $\mathrm{NaCl}$ and $\mathrm{KCl}$ indicates that this effect is not solute-specific but indeed dependent on the solute potential of the culture medium. Since sorbitol and lactose are very poorly utilized by strain $299 \mathrm{R}$, it is unlikely that their assimilation would have triggered the high levels of ipdC expression observed above.

Because the total water potential of microbial substrates is a function of both the solute and matric potentials of the substrates (Harris 1981), the effect of matric potential on ipdC expression was also investigated. Matric potential, which is negligible in liquid media, may be an important component of water potential in the microenvironment of bacteria on plant surfaces. Low matric potential induced expression of ipdC to levels similar to those induced by low solute potential. Thus, the increased transcription of ipdC under low solute and matric potentials strongly suggests that conditions of low water availability are sufficient to induce ipdC transcription. This is supported by the rapid increase in ice activity of 299MX149 upon hyperosmotic shock in minimal A medium containing
$1.28 \% \mathrm{NaCl}$. Interestingly, unlike many osmoregulated genes of $E$. coli that are induced during entry into stationary phase in culture in the absence of high salt concentrations (HenggeAronis 1996), ipdC is not growth phase-regulated since its expression remained low during all phases of growth in minimal and rich media that are low in solutes.

The finding that ipdC is transcribed much more highly on agar plates than in culture broth of the same composition is intriguing. Although other factors may be responsible for this induction, it is possible that cells growing in the dense exopolysaccharide matrix of the colony sense high matric potentials. Differential control of the expression of cell surface proteins of Staphylococcus aureus grown in liquid and solid media has also been reported (Cheung and Fischetti 1988), and the induction of swarmer cell genes (laf) in Vibrio parahaemolyticus is triggered by growth on solidified media, in PEG-amended culture broth, or under iron-limiting conditions (McCarter and Silverman 1990). Likewise, ipdC expression may be involved in the recognition of, or adaptation to, conditions related to growth on a surface, a feature common in the natural habitat of bacterial epiphytes; the response to low water availability seems logical since free water may frequently be absent in the phylloplane.

The enhanced production of TOL and IAA by strain 299R under hyperosmotic culture conditions is consistent with the observation that ipdC is osmoresponsive. The higher levels of indolepyruvate decarboxylase present under conditions of high osmolarity apparently contribute to increased activity of the IAA pathway, leading to high TOL and IAA production. It is unlikely that the increased synthesis of IAA and TOL was mediated solely by reduced growth rates associated with high osmoticum, since elevated quantities of IAA and TOL were not detected at low growth rates conferred by incubation of cultures in minimal A broth at suboptimal temperatures (Brandl et al. 1996). The failure to observe increased levels of IAA and TOL synthesis at the highest osmolarities, where ipdC transcription was high, may be attributable to the general inhibitory effect of low intracellular water activity on enzymatic functions. Additionally, posttranscriptional regulation of the components of the IAA pathway may occur and be important in IAA biosynthesis. Complementation studies of ipdC mutants showing that IAA synthesis was repressed in ipdC merodiploids of $E$. herbicola 299R and 299MX149, whereas TOL production increased, suggest that post-transcriptional regulation occurs in this pathway (Brandl and Lindow 1996).

Although ipdC was induced in conditions of low water availability, its inactivation did not affect the osmotolerance of 299MX149 in vitro since the latter exhibited growth rates comparable to those of the parental strain in media of low solute or matric potential. ipdC is therefore not essential for, and may not be involved in, adaptation to and survival under osmotic and matric stress per se. The possibility remains that ipdC is not directly under osmotic control, but that its rate of transcription is dependent on other physiological factors that are affected by osmotic stress, as was proposed for the ompC and ompF genes of Escherichia coli K-12 and Salmonella typhimurium (Csonka 1991). It is unlikely, however, that induction of ipdC was caused solely by the reduced growth rates resulting from low water potentials, since the slowing of growth of 299MX149 in nitrogen-limited minimal A medium 
did not increase the expression of ipdC. The observation that the levels of transcription of ipdC can be as high on wet as on dry bean plants (data not shown) suggested that ipdC may respond to stimuli other than osmotic stress per se, or that ipdC is part of a family of genes within a regulon that is highly active during growth on plant surfaces. Alternatively, it is possible that bacteria experience osmotic stress in their microenvironment even when the leaf surface appears moist, so that a detectable difference in ipdC expression on dry versus wet plants should not be expected.

It seems likely that strain $299 \mathrm{R}$ uses the osmotically responsive ipdC gene to trigger IAA synthesis, which may play a role in adaptation to conditions experienced on plant surfaces. Low water availability, which restricts diffusion of nutrients and microbial locomotion (Harris 1981), is most likely a condition frequently encountered by bacterial epiphytes in their microenvironment on plant surfaces. Wilson and Lindow (1994a, 1994b) have demonstrated that bacterial populations in the phyllosphere are nutrient-limited and compete for carbon sources, and for nitrogen sources when the plant itself is nitrogen-limited (M. Wilson, personal communication). Exogenously applied auxin stimulated the release of large quantities of monosaccharides and oligosaccharides from the plant cell wall (Fry 1989; Goldberg 1975). The production of IAA may thus enable bacterial epiphytes to modify their microhabitat by increasing the leakage of nutrients from plant cells in their vicinity, in particular when access to nutrients is limited due to low water availability. It is interesting to note that certain hrp genes of P. syringae pv. phaseolicola and the hrpF gene of $X$. campestris pv. campestris, two plant pathogenic bacteria that are exposed to environmental conditions within plant tissue that are nearly opposite from those encountered by epiphytic bacteria (high water potential and low $\mathrm{pH}$ ), are downregulated by high osmotic strength of the culture medium (Rahme et al. 1992; Schulte and Bonas 1992). It is possible that these two groups of plant-associated bacteria have each evolved mechanisms to induce conditional expression of genes involved in the exploitation of plant habitats by environmental cues specific for internal and external surfaces of plants, respectively. The observation that ipdC is transcriptionally activated by conditions of low water availability in culture and induced on plants is consistent with the hypothesis that IAA synthesis by epiphytic bacteria such as E. herbicola 299R may contribute to their ability to modify their habitat and exploit plant surfaces. It will be interesting to determine if other genes of the IAA pathway are also hyperosmotically inducible or if multiple signals are necessary for maximum activity of this pathway in plant-associated bacteria.

\section{MATERIALS AND METHODS}

\section{Bacterial strains and plasmids.}

The source and characteristics of Erwinia herbicola strain 299R were described previously (Brandl et al. 1996). E. herbicola strain 299MX149 is a derivative of strain 299R containing a single Tn3-spice insertion within ipdC that was introduced by homologous recombination into the chromosome (Brandl and Lindow 1996). The Tn3-spice insertion in strain 299 MX149 is fused to ipdC such that the direction of transcription of the ice nucleation gene $i n a Z$ is the same as that of ipdC. Plasmid pJEL1703 was constructed by ligation of the ice $C$ gene from $P$. syringae into plasmid pVSP61, which confers kanamycin resistance (Loper and Lindow 1994). The iceC gene was also cloned into EcoRI-cleaved plasmid pCPP8, which confers streptomycin and spectinomycin resistance (gift of David Bauer, Cornell University), to generate pCPPICE. The ice $C$ gene of $P$. syringae Cit7R 1 is very similar to the inaZ gene from another strain of this species (Loper and Lindow 1994). As shown in this study, the expression of ice nucleation from iceC is only weakly responsive to low solute and matric potentials, and to conditions on plant surfaces; thus, ice $C$ was used as a control when the regulation of ipdC was studied with ipdC-inaZ fusions. pJEL1703 and pCPPICE were introduced by triparental mating into strain 299R, in which they were stably maintained, to produce strains 299R(pJEL1703) and 299R(pCPPICE), respectively.

\section{Media and growth conditions.}

Inocula for all in vitro and in situ experiments were prepared by growing $E$. herbicola strain $299 \mathrm{R}$ or its derivatives to stationary phase in minimal A medium (Miller 1972) at $27^{\circ} \mathrm{C}$. When appropriate, the medium was supplemented with kanamycin $\left(20 \mu \mathrm{g} \mathrm{ml}^{-1}\right)$, rifampicin $\left(100 \mu \mathrm{g} \mathrm{ml}^{-1}\right)$, or streptomycin $\left(20 \mu \mathrm{g} \mathrm{ml}^{-1}\right)$. The cells were then centrifuged, washed twice in potassium phosphate buffer $(10 \mathrm{mM}, \mathrm{pH} 7.0)$, and either resuspended in the buffer for inoculation onto plants or added to the various culture media for in vitro experiments.

Bacterial cell concentrations were estimated by dilution plating appropriate dilutions of cell suspensions on LB agar plates containing appropriate antibiotics at concentrations mentioned above. When present in the medium, TRP was added at a final concentration of $0.02 \%$.

\section{Assessment of transcriptional activity.}

The level of transcriptional activity of ipdC-inaZ fusions in culture and on plant surfaces was quantified by a dropletfreezing assay (Lindow 1990) at $-9^{\circ} \mathrm{C}$. Ice nucleation activity was normalized for the concentration of bacterial cells estimated by dilution plating. All cultures tested for ice nucleation activity were incubated in $10 \mathrm{ml}$ of liquid medium containing $0.02 \%$ TRP, unless mentioned otherwise. Cells were added to the medium at a starting concentration of $5 \times 10^{6}$ cells $\mathrm{ml}^{-1}$ and incubated at $24^{\circ} \mathrm{C}$ for $24 \mathrm{~h}$ before ice nucleation activity was assayed.

For assessment of ipdC expression on solid medium, cells were spread on LB and minimal A agar at a density of ca. 200 $\mathrm{CFU}$ per plate. The plates were incubated at $24^{\circ} \mathrm{C}$ for 48 and $60 \mathrm{~h}$, respectively. The cells were then suspended in potassium phosphate buffer (10 mM, pH 7.0), and tested for ice nucleation activity at $-9^{\circ} \mathrm{C}$. Anaerobic conditions were obtained by incubation of inoculated plates at $24^{\circ} \mathrm{C}$ in a BBL GasPak anaerobic jar containing a hydrogen and carbon dioxide generator and a GasPak anaerobic indicator strip (Becton Dickinson, Cockeysville, MD). The plates were incubated for 6 days and the cells then assayed for ice nucleation activity.

The solute potential of basal minimal A medium was estimated to be $-0.7 \mathrm{Mpa}$. Its solute potential was adjusted to $-1.2,-1.7,-2.7$, and $-3.7 \mathrm{Mpa}$ by the addition of $\mathrm{NaCl}$ at a final concentration of $0.64,1.28,2.57$, and $3.85 \%$, respectively, or by the addition of $\mathrm{KCl}$, sorbitol, or $\beta$-lactose, at appropriate concentrations based on those cited in the Handbook of Chemistry and Physics (Weast 1978). A matric potential of 
$0,-0.2,-0.5$, and $-0.75 \mathrm{Mpa}$ in minimal A cultures was achieved by the addition of PEG 4000 to minimal A medium at a concentration of $0,6,13$, and $16 \%$, respectively.

\section{Quantification of TOL and IAA production in culture.}

Strain $299 \mathrm{R}$ was incubated $48 \mathrm{~h}$ at $27^{\circ} \mathrm{C}$ in $50 \mathrm{ml}$ of minimal A medium containing $0.02 \%$ TRP, and $0,0.64,1.28$, or $2.57 \% \mathrm{NaCl}$. TOL and IAA were extracted from the culture supernatant and quantified by high-performance liquid chromatography as previously described (Brandl et al. 1996). The quantity of TOL and IAA measured was normalized for the number of cells present in each culture, which was estimated by dilution plating on LB agar amended with rifampicin.

\section{Evaluation of bacterial populations and ipdC expression on plant tissue.}

Dormant potted young pear trees (cv. Comice grafted on quince rootstock) were placed in the greenhouse in late winter to promote bloom. The average minimum and maximum temperatures in the greenhouse were 20 and $35^{\circ} \mathrm{C}$, respectively; average minimum and maximum relative humidities were 30 and $60 \%$, respectively. Trees were inoculated at ca. $90 \%$ bloom by spraying the blossoms with strain 299R, 299MX149, or 299R(pJEL1703) at $10^{6}$ cells $\mathrm{ml}^{-1}$ until run-off. Inoculum was prepared as described above. Each treatment was applied to two replicate trees. Twenty and 10 flowers from trees treated with either strain 299R or 299MX149, and with strain 299R(pJEL1703), respectively, were sampled at regular time intervals from inoculation until senescence. The flowers were placed in $5 \mathrm{ml}$ of sterile phosphate buffer in a small plastic bag, sonicated for $7 \mathrm{~min}$, homogenized by hand, and dilution plated on LB agar containing rifampicin, streptomycin, or kanamycin, as appropriate, for estimation of the population size of the inoculated strain. The homogenates were also tested for ice nucleation activity by the dropletfreezing assay.

Strains 299R, 299MX149, and 299R(pJEL1703) were inoculated onto young greenhouse-grown tobacco (Nicotiana tabacum cv. Turkish) and bean (Phaseolus vulgaris cv. Bush Blue Lake 274) plants, at a concentration of $10^{7}$ and $10^{3}$ cells $\mathrm{ml}^{-1}$, respectively. Each plant was covered with a plastic bag and placed in a laboratory at room temperature. Five and 10 leaves, respectively, were sampled from five replicate plants at regular time intervals, and placed in 40 and $20 \mathrm{ml}$ of sterile phosphate buffer, respectively. Leaves were sonicated for 7 min and the suspension vigorously agitated; appropriate dilutions were then plated on LB agar supplemented with appropriate antibiotics for estimation of bacterial populations. Leaf washings were assayed also for ice nucleation activity as described above.

\section{LITERATURE CITED}

Brandl, M., Clark, E. M., and Lindow, S. E. 1996. Characterization of the indole-3-acetic acid (IAA) pathway in an epiphytic strain of $\mathrm{Er}$ winia herbicola and IAA production in vitro. Can. J. Microbiol. 42: 586-592.

Brandl, M. T., and Lindow, S. E. 1996. Cloning and characterization of a locus encoding an indolepyruvate decarboxylase involved in indole-3- acetic acid synthesis in Erwinia herbicola. Appl. Environ. Microbiol. 62:4121-4128.

Cheung, A., and Fischetti, V. 1988. Variation in the expression of cell wall proteins of Staphylococcus aureus grown on solid and liquid media. Infect. Immun. 56:1061-1065.

Cirvilleri, G., and Lindow, S. E. 1994. Differential expression of genes of Pseudomonas syringae on leaves and in culture evaluated with random genomic lux fusions. Mol. Ecol. 3:249-257.

Csonka, L. N. 1991. Prokaryotic osmoregulation: Genetics and physiology. Annu. Rev. Microbiol. 45:569-606.

Fry, S. C. 1989. Cellulases, hemicelluloses and auxin-stimulated growth: A possible relationship. Physiol. Plant. 75:532-536.

Goldberg, R. 1975. Cell wall polysaccharidase activities and growth processes: A possible relationship. Physiol. Plant. 50:261-264.

Harris, R. F. 1981. Effect of water potential on microbial growth and activity. Pages 23-95 in: Water potential relations in soil microbiology. vol. J. F. Parr, ed. ASA Spec. Pub. No. 9. Soil Soc. Am., Madison, WI.

Hengge-Aronis, R. 1996. Back to $\log$ phase: $\delta^{\mathrm{s}}$ as a global regulator in the osmotic control of gene expression in Escherichia coli. Mol. Microbiol. 21:887-893.

Huynh, T. V., Dahlbeck, D., and Staskawicz, B. J. 1989. Bacterial blight of soybean: regulation of a pathogen gene determining host cultivar specificity. Science 245:1374-1376.

Lindgren, P. B., Frederick, R., Govindarajan, A. G., Panopoulos, N. J., Staskawicz, B. J., and Lindow, S. E. 1989. An ice nucleation reporter gene system: Identification of inducible pathogenicity genes in $\mathrm{Pseu}$ domonas syringae pv. phaseolicola. EMBO J. 8:1291-1301.

Lindow, S. E. 1987. Severity of pear fruit russeting associated with epiphytic indoleacetic acid-producing bacteria. Phytopathology 77:1724.

Lindow, S. E. 1990. Bacterial ice nucleation measurements. Pages 428434 in: Methods in Phytobacteriology. D. C. Sands, Z. Klement, and K. Rudolf, eds. Akadémiai Kiadó, Budapest.

Lindow, S. E. 1995. The use of reporter genes in the study of microbial ecology. Mol. Ecol. 4:555-566.

Loper, J. E., and Lindow, S. E. 1994. A biological sensor for iron available to bacteria in their habitats on plant surfaces. Appl. Environ. Microbiol. 60:1934-1941.

McCarter L., and Silverman, M. 1990. Surface-induced swarmer cell differentiation of Vibrio parahaemolyticus. Mol. Microbiol. 4:10571062.

Miller, J. H. 1972. Experiments in Molecular Genetics. Cold Spring Harbor Laboratory, Cold Spring Harbor, New York.

Morgan, J. V., and Tukey, H. B., Jr. 1964. Characterization of leachate from plant foliage. Plant Physiol. 39:590-593.

Rahme, L. G., Mindrinos, M. N., and Panopoulos, N. J. 1992. Plant and environmental sensory signals control the expression of hrp genes in Pseudomonas syringae pv. phaseolicola. J. Bacteriol. 174:3499-3507.

Sambrook, J., Fritsch, E. F., and Maniatis, T. A. 1989. Molecular Cloning: A Laboratory Manual. 2nd ed. Cold Spring Harbor Laboratory, Cold Spring Harbor, New York.

Schulte, R., and Bonas, U. 1992. A Xanthomonas pathogenicity locus is induced by sucrose and sulfur-containing amino acids. Plant Cell 4: 79-86.

Weast, R. C. 1978. Handbook of Chemistry and Physics. 8th ed. CRC, West Palm Beach, FL.

Weibull, J., Ronquist, F., and Brishammar, S. 1990. Free amino acid composition of leaf exudates and phloem sap. Plant Physiol. 92:222226

Wilson, M., and Lindow, S. E. 1993. Effect of phenotypic plasticity on epiphytic survival and colonization by Pseudomonas syringae. Appl. Environ. Microbiol. 53:410-416.

Wilson, M. and Lindow, S. E. 1994a. Ecological similarity and coexistence of epiphytic ice-nucleating $\left(\right.$ ice $\left.^{+}\right)$Pseudomonas syringae strains and a non-ice-nucleating (ice) biological control agent. Appl. Environ. Microbiol. 60:3128-3137.

Wilson, M. and Lindow, S. E. 1994b. Coexistence among epiphytic bacterial populations mediated through nutritional resource partitioning. Appl. Environ. Microbiol. 60:4468-4477.

Yamada, T. 1993. The role of auxin in plant-disease development. Annu. Rev. Phytopathol. 31:253-273. 